Results Of the planned 1400 MSM, 99 (53 HIV +) have been recruited. Two equivocal results were excluded from analysis. Some of the questionnaire data were missing. 80\%(73/91) were symptomatic and $60 \%$ (57/96) reported unprotected anal sex in the last month. The prevalence of CT and/or NG infection was 35\% (95\% CI 26\% to 45\%), CT alone $14 \%$ (95\% CI $8 \%$ to $23 \%$ ) and NG alone $21 \%$ (95\% CI $13 \%$ to $30 \%)$. The sensitivity and specificity of PS vs SOC to detect CT/NG is $88 \%(95 \%$ CI $72 \%$ to $96 \%$ ) and $100 \%$ (95\% CI $93 \%$ to $100 \%$ ), respectively (abstract P98 table 1). PS failed to detect four NG cases (3 pharynx, 1 rectum). MSM reported confidence $(n=74,86 \%)$ and willingness $(\mathrm{n}=75,88 \%)$ to take their own samples (see abstract P98 table 1).

Abstract P98 Table 1 Sensitivity and specificity of pooled samples according to test

\begin{tabular}{lllll}
\hline & $\begin{array}{l}\text { Number } \\
\text { positive (\%) SOC } \\
\text { testing [95\% Cl] }\end{array}$ & $\begin{array}{l}\text { Number } \\
\text { positive (\%) } \\
\text { PS testing }\end{array}$ & $\begin{array}{l}\text { Sensitivity \% } \\
{[95 \% \text { CI] }}\end{array}$ & $\begin{array}{l}\text { Specificity \% } \\
{[95 \% \text { CI] }}\end{array}$ \\
\hline $\begin{array}{l}\text { CT \&/ or NG* } \\
\text { (*CT\&NG, } \mathrm{n}=4)\end{array}$ & $34(35)[26$ to 45$]$ & $30(88)$ & $88[72$ to 96$]$ & $100[93$ to 100] \\
CT & $14(14)[8$ to 23$]$ & $14(100)$ & $100[73$ to 100$]$ & $100[95$ to 100] \\
NG & $20(21)[13$ to 30$]$ & $16(80)$ & $80[56$ to 93$]$ & $100[94$ to 100$]$ \\
\hline
\end{tabular}

Discussion Pooling specimens in MSM offers the potential for significant savings and improved access to testing. Missed infections may be due to sampling error or low organism load. The evaluation of this strategy continues.

\section{P99 SOCIO-DEMOGRAPHIC AND BEHAVIOURAL CHARACTERISTICS OF MEN WHO HAVE SEX WITH MEN (MSM) AND HETEROSEXUALS INFECTED WITH GONORRHOEA}

doi:10.1136/sextrans-2012-050601c.99

C Obi, ${ }^{*}$ S Chisholm, E Webster, K Town, J Anderson, T Nichols, C Ison, C Lowndes. Health Protection Agency, London, UK

Background As well as monitoring antimicrobial resistance, the enhanced Gonococcal Resistance to Antimicrobial Surveillance Programme dataset can be used to understand the epidemiology of gonococcal infection.

Objective To explore socio demographic and behavioural characteristics of MSM (HIV positive and negative) and heterosexuals (male and female) infected with gonorrhoea.

Methods Demographic and behavioural data from Gonococcal Resistance to Antimicrobial Surveillance Programme, collected annually between July and September 2005-2010 from 26 sentinel GUM clinics were analysed.

Results Of 9239 gonorrhoea cases, 3089 (36\%) were in MSM, of whom $861(28 \%)$ were HIV positive; 5588 in heterosexuals, of whom 3012 (54\%) were men. Predominantly of white ethnic background, HIV positive MSM (mean age $36 \mathrm{y}$ ) were older than HIV negative MSM (mean age $30 \mathrm{y}$ ). A higher proportion of HIV positive than negative MSM were co-infected with another STI $(\mathrm{OR}=1.6,95 \%$ CI 1.3 to 1.9$)$, mainly chlamydia (18\% vs $15 \%)$ or syphilis ( $5 \%$ vs $2 \%$ ). HIV positive MSM were also more likely $(\mathrm{OR}=1.5$, CI 1.3 to 1.8$)$ to report rectal gonococcal infection. Over a quarter of HIV positive MSM reported $\geq 6$ sexual partners in the past 3 months compared to $18 \%$ of HIV negative MSM. Within the heterosexual population, higher proportion of women than men were of white ethnic background (74\% vs $43 \%)$ and $<25$ y $(72 \%$ vs $47 \%$ ). Compared to heterosexual men, women were more likely to be co-infected with another STI ( $O R=1.5$, CI 1.4 to 1.7$)$ primarily chlamydia (41\% vs $35 \%$ ). Nearly two-third of heterosexual men reported $\geq 2$ sexual partners in past 3 months while most women $(64 \%)$ reported one or no sexual partners.
Conclusion Gonorrhoea is concentrated amongst specific population sub-groups. Our analysis indicates that these groups are at high risk of contracting and transmitting other STIs as well as HIV, and underlines the need for targeted interventions.

\section{P100 \\ ASSESSMENT OF BACTERIAL SEXUALLY TRANSMITTED INFECTION (STI) SCREENING FOLLOWING SEXUAL ASSAULT}

doi:10.1136/sextrans-2012-050601c.100

S Wilson, ${ }^{*}$ D Wardle, G Coia. Sandyford

Background Chlamydia trachomatis and Neiserria gonorrhoea screening following sexual assault is undertaken primarily for sexual health purposes but can potentially facilitate criminal investigation in cases of penetration at sexually naive sites. Antibiotic prophylaxis at first attendance (within 7 days of the assault) is not routine but may be given when the return for screening is unlikely.

Aim To improve bacterial STI screening and management in complainers attending our sexual assault referral centre (SARC).

Objectives To determine if complainers were adequately screened for bacterial STIs and if communication with health professionals regarding repeat screening was adequate.

To review prophylactic antibiotic use.

To review the forensic significance of STI screening.

To identify factors which may improve the uptake of screening. Methods 100 case records were reviewed and information relevant to our objectives extracted. Six cases were excluded.

Results $81 \%$ had a STI screen taken at presentation. Only $13 \%$ returned for repeat screen after incubation, confirming Chlamydia in two cases. All but one repeat screen correlated to the site of exposure. GPs were informed of the need for a repeat screen in $74 \%$ of cases. 59\% had an alert sited on their sexual health record highlighting the need for a repeat screen. Antibiotic prophylaxis was given in 25 cases with reasons documented in only 4.

Discussion and/or Conclusion STI screening post sexual assault may be improved through better communication with complainers and other healthcare providers. Improvements to communication methods and training are required to facilitate this. The concern of emerging gonococccal resistance should be considered prior to administering prophylactic antibiotics. One individual, in whom there was no previous sexual contact and baseline screen was negative, had Chlamydia on repeat sample. This may be supportive of the assailant as the source of infection, indicative of the potential forensic role of STI screening.

\section{P101 OLYMPIC OUTREACH: STI TESTING FOR CONSTRUCTION WORKERS}

doi:10.1136/sextrans-2012-050601c.101

S Shanmugaratnam, ${ }^{*}$ P Horne, K M Coyne. Homerton University Hospital, London, UK

Background It was feared that construction of venues for the 2012 Olympic and Paralympics Games would increase the burden of sexual ill-health in East London, due to a surge in migrant construction workers and commercial sex work.

Aims/Objectives We analysed data from outreach to construction sites at the Olympic Park and Village in Stratford, East London. We reported demographics, sexual risk factors and STI rates.

Methods An outreach team visited the Olympic site between February 2009 and October 2011. Clients completed a triage form about symptoms and sexual risk factors. Clients were offered nucleic acid amplification tests for Gonorrhoea and Chlamydia, using urine samples from men, and self-taken vulvovaginal swabs 
from women. Serology was offered for HIV and Syphilis. Hepatitis B serology was offered to those who reported intravenous drug use or sex with commercial sex workers. Anyone diagnosed with an STI was offered treatment at Occupational Health services on site or a local sexual health clinic.

Results In total 614 clients were seen, with a median age of 28 years. $91 \%$ were men. There were 19 different ethnic groups. Nearly half (285/614, 46\%) reported their ethnic group as English/Scottish/ Welsh. The other large ethnic groups were 53 Indian (9\%), 52 Eastern European (8\%) and 39 Irish (6\%). 20 clients (3\%) had Chlamydia and one was diagnosed with Hepatitis B. Reported levels of sexual risk factors are shown in abstract P101 table 1.

Abstract P101 Table 1 Sexual risk factors of in the olympic site construction workers

\begin{tabular}{ll}
\hline Sexual risk factor & No. of people/ \% of sample \\
\hline Sex between men & $5(<1 \%)$ \\
Previous STI & $20(3 \%)$ \\
More than one sexual partner in last 3 months & $173(28 \%)$ \\
Intravenous drug use & $3(<1 \%)$ \\
Use of commercial sex workers & $3(\mathrm{n}=76)(4 \%)$ \\
\hline
\end{tabular}

Conclusions There was a low prevalence of STIs in these construction workers, contradicting prior fears of a high disease burden. Reported levels of sexual risk factors, including use of commercial sex workers, were low.

\section{P102 A HIGHLY COST-EFFECTIVE AND TARGETED SERVICE PROMOTION CAMPAIGN USING THE SOCIAL MEDIA SITE FACEBOOK}

doi:10.1136/sextrans-2012-050601c.102

\section{S Day, A Hughes.* Chelsea and Westminster Hospital, London, UK}

Background Facebook is the leading social media site in the world with over 30 million active users in the UK, $50 \%$ of who log on every day. It enables promotion to be targeted to specified demographic groups and is cost-effective because advertisers only pay for users who click through to their website. Using Facebook we led an advertising campaign to men having sex with men (MSM).

Method The advert featured from 9th October to 31st December 2009 and offered a free rapid HIV testing and sexual health screening service to users actively logged on to their Facebook account. We targeted men interested in men, aged between 18 and $50 \mathrm{yrs}$, that were single or in a relationship and living within 50 miles of our city. Users clicking onto the advert were automatically diverted to our website's appointments section. Each click-through incurred a small fee ("Pay-per-click").

Results The campaign targeted 30580 registered Facebook MSM users. Over 3 months the advert appeared 2.86 million times. Up to 9750 individuals were exposed to the advert on any given day and it frequently featured several times a day to each user. In total, 872 $(2.9 \%)$ registered MSM users clicked through to our website at an average cost of $£ 0.55$ per click. These were aged 18-24 (55\%), 25-34 (27\%), 35-44 (14\%) and 45-54 (4\%) years. The total cost of the campaign was $£ 483$. Facebook provided regular statistical reports which enabled us to monitor its effect, control our budget and prospectively tailor the advert accordingly.

Discussion This simple Pay-per-click Facebook campaign advertised to $8.5 \%$ of the UK MSM population (estimated UK MSM population is 3.6 million). Using Payment by Results income, only $0.3 \%$ (3-4) of clickers would need to attend our service to make the campaign costneutral. Whilst the functionality, diversity and social reach of Face- book is growing year on year, this presents a highly cost-effective opportunity for service/health promotion to hard to reach groups.

\section{Electronic patient records and use of IT P103 ENHANCING PATIENT SAFETY IN A LARGE HIV OUT- PATIENT SERVICE: EVALUATION OF AN ELECTRONIC RESULTS CHECKING SYSTEM FOR BLOOD TESTS}

doi:10.1136/sextrans-2012-050601c.103

D Nugent, ${ }^{*}$ N Uthayakumar, R Ferrand, S Edwards, P Benn. Mortimer Market Centre, London, UK

Background HIV services face the challenge of regular monitoring of a growing patient cohort and ensuring prompt action upon abnormal results. We identified a number of issues using a paperbased results system (PBS) including: (1) missing results, (2) delayed delivery, (3) clinician error, (4) lack of audit trail, placing patients at risk of delayed identification of drug toxicity and serious conditions for example, acute hepatitis.

Aims We piloted an electronic results checking system (ERC) which classifies results as normal or abnormal (non-urgent (NUAbn) or urgent (UAbn)) to compare the speed and performance of PBS and ERC in identifying biochemical abnormalities.

Methods Between 4 July 2011 and 22 July 2011 we compared the time intervals from sampling to (1) receipt of results; (2) clinician review of UAbn/NUAbn and (3) review of NUAbn by a clinician. Abnormalities were graded and both systems reviewed daily. Data were analysed using STATA V.11.0. Mann-Whitney U tests were used to compare the intervals.

Results Of 513 patients undergoing $\geq$ one blood test, $296(57.7 \%)$ had $\geq$ one biochemical abnormality identified by the ERC $(10.7 \%$ UAbn, $42.3 \%$ NUAbn and $47 \%$ not clinically significant). Of these, PBS simultaneously identified $83 \%$. The median interval between sampling to (1) receipt of results was 1 (IOR $1-2$ ) vs 4 days (IOR 3-5), $\mathrm{p}<0.0001$; (2) clinician review 3 (IOR 1-4) vs 3 (IOR 3-6) days, $\mathrm{p}<0.037$; and (3) review of NUAbn by clinician 2 (IOR $1-4$ ) vs 10 days (IOR 9-12), $\mathrm{P}=0.136$, for ERC and $\mathrm{PBS}$ respectively. $11 \%$ of the missing PBS results were classified UAbn. ERC missed three abnormalities highlighting a software error which has now been corrected.

Conclusion We demonstrate the use of IT to review blood results leads to the faster identification of biochemical abnormalities, which are common in our HIV cohort, facilitating their timely management. We anticipate the use of ERC in routine practice will avoid delay/non-identification of a significant number of abnormal results within our service.

\section{P104 PATIENT VIEWS ON CONFIDENTIALITY IN THE ELECTRONIC ERA}

doi:10.1136/sextrans-2012-050601c.104

E Stachow, ${ }^{*}$ A Wong, Z Soogun-Shah, S Barrett. Birmingham Heartlands Hospital

Background Confidentiality is a cornerstone of good medical practice. However, sharing of medical information is often essential for safe, effective care. The advent of computerised records has increased the scope for efficient data transfer. However, the same principles for patient confidentiality remain for this format. Ambiguity in current guidelines exists regarding patient data relating to sexual health. Peer-reviewed research reveals a lack of patient understanding on confidentiality, and concerns on inappropriate data disclosure within local areas. 